\title{
A New Approach for Nonlinear Distortion Correction in Endoscopic Images Based on Least Squares Estimation
}

\author{
K. Vijayan Asari, Member, IEEE, Sanjiv Kumar, and D. Radhakrishnan,* Member, IEEE
}

\begin{abstract}
Images captured with a typical endoscope show spatial distortion, which necessitates distortion correction for subsequent analysis. In this paper, a new methodology based on least squares estimation is proposed to correct the nonlinear distortion in the endoscopic images. A mathematical model based on polynomial mapping is used to map the images from distorted image space onto the corrected image space. The model parameters include the polynomial coefficients, distortion center, and corrected center. The proposed method utilizes a line search approach of global convergence for the iterative procedure to obtain the optimum expansion coefficients. A new technique to find the distortion center of the image based on curvature criterion is presented. A dual-step approach comprising token matching and integrated neighborhood search is also proposed for accurate extraction of the centers of the dots contained in a rectangular grid, used for the model parameter estimation. The model parameters were verified with different grid patterns. The distortion-correction model is applied to several gastrointestinal images and the results are presented. The proposed technique provides high-speed response and forms a key step toward online camera calibration, which is required for accurate quantitative analysis of the images.
\end{abstract}

Index Terms-Camera calibration, distortion correction, endoscopy, expansion polynomial.

\section{INTRODUCTION}

$\mathbf{M}$ INIMALLY invasive therapy (MIT) is increasingly becoming popular because of the use of natural or artificial orifices of the body for surgical procedures, which minimizes the destruction of healthy organs and tissues. Electronic videoendoscopy has become one of the commonly accepted forms of diagnostic and therapeutic procedures, due to the advent of miniature CCD cameras and associated microelectronics. Videoendoscopes facilitate observation, documentation, and electrical manipulation of the images of the internal structure of the gastrointestinal tract. In these endoscopes, cameras with a wide viewing angle lens (fish-eye lens) are used to enhance the imaging capability, which permits the capturing of larger field in a single image [1]. However, it has been noted that the images obtained from electronic endo-

Manuscript received June 1, 1998; revised January 4, 1999. The Associate Editor responsible for coordinating the review of this paper and recommending its publication was W. Higgins. Asterisk indicates corresponding author.

K. V. Asari is with the Center for High Performance Embedded Systems, Nanyang Technological University, Singapore-639798.

S. Kumar is with the Robotics Institute, Carnegie Mellon University, Pittsburgh, PA 15213 USA.

*D. Radhakrishnan is with the Division of Computing Systems, Nanyang Technological University, Nanyang Avenue, Singapore-639798.

Publisher Item Identifier S 0278-0062(99)04499-7. scopes show barrel-type spatial distortion, due to wide-angle configuration of the camera lens. Barrel distortion introduces nonlinear changes in the image, due to which, image areas near the distortion center are compressed less, while areas farther from the center are compressed more. Because of this, the outer areas of the image look significantly smaller than their actual size. This inhomogeneous image compression introduces significant errors in the results obtained during feature extraction. Continuous estimation of quantitative parameters, such as area and perimeter, is of considerable importance while performing clinical endoscopy. Unless the distortion is corrected, estimation errors could be very large [2]-[4]. In addition, the distortion causes complications while using token matching techniques for pattern recognition. Distortion correction is also a prerequisite for the camera calibration to obtain extrinsic and intrinsic camera parameters [5], [6].

Several researchers have presented various mathematical models of the image distortion and techniques to find the model parameters to complete the distortion-correction procedure. Tsai [7] proposed a radial lens distortion model which describes a two-dimensional (2-D) image-correction technique. A prism-distortion model was used in [8] to correct the tangential distortion in an image. Nomura et al. [9] presented a calibration technique for high-distortion TV camera lenses. However, this method requires precise placement of the calibration chart. Thus, a small shift of the chart prompts considerable errors in distortion correction. Weng [10] has explained radial, decentering, and thin prism-types of distortions and techniques to model them mathematically. All the above models give reasonable results for images obtained from cameras with normal viewing objective lenses, but these models are not effective for electronic endoscopes which use wide-angle lens cameras. Smith et al. [11] gave a formulation in which distortion was assumed to be purely radial, and orthogonal Chebyshev polynomials were used to determine the model parameters. Hideaki et al. [12] presented a different method for estimation of the model parameters, in which a moment matrix was obtained from a set of image points, and distorted grid lines in the image were straightened on the basis of the smallest characteristic root of the moment matrix.

In this paper, we use a similar distortion-correction formulation, based on polynomial mapping as used by Hideaki. We propose a new technique based on least squares estimation to obtain the coefficients of the correction polynomial. This approach is simpler and faster than the one used by Hideaki. Furthermore, a method for accurate determination of the critical points of calibration grid, based on a dual-step approach 
is also presented. The proposed technique is independent of the orientation of the calibration chart. Thus, it does not require precise placement of the chart and the placement errors in distortion-correction formulation can be avoided. The performance of the proposed distortion-correction algorithm is validated with grid patterns of different orientations. For the precise correction of images, the distances of the object points from the camera in three-dimensional (3-D) space must be included in the distortion-correction procedure. However, the method proposed here forms a key step toward the precise distortion correction of the endoscopic images.

\section{THEORETICAL MODEL}

The distortion-correction technique presented here assumes that the distortion is radial about the distortion center. Although nonlinear magnification of the distorted endoscopic image in two dimensions is needed to correct the barrel distortion, the assumption precludes the loss of generality as a typical endoscope lens is circularly symmetric within narrow precision limits [11]. This assumption simplifies the model by converting a 2-D distortion problem into a onedimensional (1-D) problem. Let the distorted and corrected (or undistorted) image spaces are represented by $\left(U^{\prime}, V^{\prime}\right)$ and $(U, V)$, respectively, and the distortion center and the corrected center by $\left(u_{c}^{\prime}, v_{c}^{\prime}\right.$ and $\left(u_{c}, v_{c}\right)$. The distortion center $\left(u_{c}^{\prime}, v_{c}^{\prime}\right)$ is a point in the distorted image space such that the straight lines in the object space passing through it remain straight in the image space. The corrected center $\left(u_{c}, v_{c}\right)$ is a point in the corrected image space about which the expansion of distorted image gives a final corrected image. In the distorted image space, magnitude $\rho^{\prime}$ of a vector $\boldsymbol{P}^{\prime}$ from the distortion center to any pixel location $\left(u^{\prime}, v^{\prime}\right)$ and the angle $\theta^{\prime}$ made by this vector from the horizontal $U^{\prime}$-axis are given by

$$
\rho^{\prime}=\sqrt{\left(u^{\prime}-u_{c}^{\prime}\right)^{2}+\left(v^{\prime}-v_{c}^{\prime}\right)^{2}} \quad \theta^{\prime}=\arctan \left(\frac{v^{\prime}-v_{c}^{\prime}}{u^{\prime}-u_{c}^{\prime}}\right) .
$$

Let the same pixel be assigned to a new location $(u, v)$ in the corrected image space and the magnitude $\rho$ and argument $\theta$ of the corresponding vector $\boldsymbol{P}$ drawn from the corrected center to the new pixel location are

$$
\rho=\sqrt{\left(u-u_{c}\right)^{2}+(v-v)^{2}} \quad \theta=\arctan \left(\frac{v-v_{c}}{u-u_{c}}\right) .
$$

The objective of the mathematical model is to obtain a relation between the vectors $\boldsymbol{P}^{\prime}$ and $\boldsymbol{P}$. An expansion polynomial of degree $N$ is defined to relate the magnitudes of the two vectors in distorted and corrected images as

$$
\rho=\sum_{n=1}^{N} a_{n} \rho^{\prime n}
$$

where $a_{n}$ 's are the expansion coefficients. As the distortion has been assumed to be purely radial, there will be no change in the arguments of the corresponding vectors $\boldsymbol{P}^{\prime}$ and $\boldsymbol{P}$, i.e., $\theta^{\prime}=\theta$. After obtaining the magnitude of the new vector, the new pixel location in the corrected image space can be calculated as

$$
u=u_{c}+\rho \cos \theta^{\prime}, \quad v=v_{c}+\rho \sin \theta^{\prime} .
$$

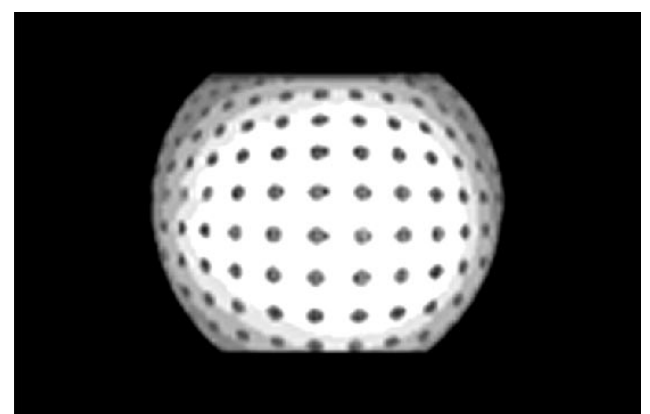

Fig. 1. Distorted image of the experimental grid of dots (diameter $-1 \mathrm{~mm}$, center distance $-2.5 \mathrm{~mm}$ ) fixed at a distance of $10 \mathrm{~mm}$ from the endoscope camera.

To map each pixel from the distorted image space onto the corrected image space, there are $N+4$ unknowns, viz., $N$ expansion coefficients $\left(a_{n}\right.$ 's), distortion center $\left(u_{c}^{\prime}, v_{c}^{\prime}\right)$, and corrected center $\left(u_{c}, v_{c}\right)$. The estimation of the distortion center and the corrected center is explained in Section II-C.

\section{A. Estimation of Expansion Coefficients}

The expansion coefficients are estimated on the basis of the degree of straightness of the points, which lie on a straight line before imaging. These are estimated in the distorted image space by straightening the grid lines of a distorted grid image. For this purpose, an experimental grid is used which contains test dots arranged in horizontal and vertical grid lines. A typical distorted image of the grid is shown in Fig. 1. A new dual-step technique for accurate computation of the test dot centers is explained in Section III. Let $P_{i j}$ denote the center of a test dot lying in the $i$ th row and $j$ th column of the grid with its coordinates at $\left(x_{i j}, y_{i j}\right)$. Let there be $L$ columns of test dots in the grid image with $k_{j}$ dot centers in the $j$ th column. A set $S_{j}$, consisting of test dot centers of the $j$ th column is defined as

$$
S_{j}=\left\{P_{1 j}, P_{2 j}, \cdots, P_{k_{j} j}\right\}, \quad \text { for } \quad j=1,2, \cdots, L .
$$

To obtain a best fit polynomial curve for each set $S_{j}$ a polynomial of degree $M$ is defined as

$$
R_{j}(x)=\sum_{\alpha=0}^{M} b_{\alpha j} x^{\alpha}
$$

To estimate the coefficients $b_{\alpha j}$ 's, least squares estimation is used which provides sufficient emphasis on all those points which are far from the approximation, without allowing them to dominate [13]. The unknowns $b_{\alpha j}$ 's are chosen to minimize the function $F_{j}$ which is defined as

$$
F_{j}=\left(\sum_{i=1}^{k_{j}}\left(y_{i j}-\sum_{\alpha=0}^{M} b_{\alpha j} x_{i j}^{\alpha}\right)^{2}\right)^{1 / 2} .
$$

Hence, $b_{\alpha j}$ 's can be calculated from

$$
\frac{\partial F_{j}}{\partial b_{\alpha j}}=0, \quad \text { for } \quad \alpha=0,1, \cdots, M .
$$

For every set $S_{j}$ from (6) $M+1$ simultaneous equations are obtained, which can be represented in a matrix form as

$$
H \boldsymbol{b}=\boldsymbol{z}
$$


where

$$
\begin{aligned}
\boldsymbol{H} & =\left[h_{l s}\right]_{(M+1) \times(M+1)} \\
h_{l s} & =\sum_{i=1}^{k_{j}} x_{i j}^{l+s}, \quad \text { for } \quad l, s=0,1, \cdots, M \\
\boldsymbol{z} & =\left[z_{0}, z_{1}, \cdots, z_{M}\right]^{t}, \quad z_{s}=\sum_{i=1}^{k_{j}} y_{i j} x_{i j}^{s} \\
\boldsymbol{b} & =\left[b_{0 j}, b_{1 j}, \cdots, b_{M j}\right]^{t} .
\end{aligned}
$$

To find the best linear fit for the set of $k_{j}$ points in $j$ th column of the test dots, a first-degree polynomial is obtained from (6). Hence, two optimum polynomial coefficients are computed using (9). A normalized error function $e_{j}$ is defined as the normalized sum of magnitudes of the perpendiculars drawn from each of the $k_{j}$ points on the best linear fit of $j$ th column as

$$
e_{j}=\frac{1}{k_{j}} \sum_{i=1}^{k_{j}}\left|\frac{b_{1 j} x_{i j}-y_{i j}+b_{0 j}}{\left(1+b_{1 j}^{2}\right)^{1 / 2}}\right| .
$$

The total error for the whole grid image is obtained by

$$
E=\sum_{j=1}^{L} e_{j}
$$

In the ideal condition, when there is no distortion in the image, the total error $E$ is zero since all grid lines will be imaged as straight lines. However, due to image distortion, $E$ has a positive value which decreases monotonously as the distortion reduces. The main objective of the mathematical model is to find the expansion coefficients $a_{n}$ 's to minimize the total error $E$. Minimization of $E$ is carried out by an iterative procedure in which the new coordinates of the test dot centers in the distorted grid image are calculated by using a new set of expansion coefficients. The recursive relationship to find the new set of expansion coefficients is derived from the line search method of guaranteed convergence [14]. This method is based on a globalization strategy to select the new coefficients. The search direction in this strategy is different from that derived from the Taylor series, as the Taylor series provides local approximation of a function. The global convergence ensures convergence of a series from any starting point to a stationary point. Line search methods are widely used for the purpose of global convergence. On the basis of this method, the expansion coefficients can be obtained by using the following recursive relationship:

$$
\begin{gathered}
a_{n}(\Delta+1)=a_{n}(\Delta)+\alpha n^{\beta} E(\Delta) \frac{1}{\left(\frac{\partial E(\Delta)}{\partial a_{n}}\right)}, \\
\text { for } n=1, \cdots N
\end{gathered}
$$

where $\alpha$ is the convergence rate parameter, $\beta$ is the expansion index, and $\partial E / \partial a_{n}$ is the error gradient. Here, $\alpha$ is chosen to ensure that for every $(\Delta+1)$ th iteration, $E(\Delta+1)<E(\Delta)$. If $\alpha$ is large, the decrease in total error $E$ predicted by the linear approximation may greatly differ from the actual decrease, and the global convergence could be violated. On the other hand, if $\alpha$ is too small, the time taken for convergence may be too long. The value of $\beta$ controls the overall expansion of the distorted image and facilitates the generation of the weights for each of the $N$ coefficients. The iterative relationship given in (12) also conforms with the principle of diminishing convergence, which ensures fast convergence at the initial phase of the correction procedure when $E$ is significantly large. The error gradient for every $\Delta$ th iteration can be computed using (13), shown at the bottom of this page. To obtain $\partial b_{0 j} / \partial a_{n}$ and $\partial b_{1 j} / \partial a_{n}, b_{0 j}$ and $b_{1 j}$ are obtained from (9) as

$$
\left[\begin{array}{l}
b_{0 j} \\
b_{1 j}
\end{array}\right]=\left[\begin{array}{cc}
k_{j} & \sum_{i} x_{i j} \\
\sum_{i} x_{i j} & \sum_{i} x_{i j}^{2}
\end{array}\right]^{-1}\left[\begin{array}{c}
\sum_{i} y_{i j} \\
\sum_{i} x_{i j} y_{i j}
\end{array}\right]
$$

where $\Sigma_{i}$ denotes $\Sigma_{i=1}^{k_{j}}$. The derivatives $\partial b_{0 j} / \partial a_{n}$ and $\partial b_{1 j} / \partial a_{n}$ can be calculated as

$$
\begin{aligned}
\frac{\partial b_{0 j}}{\partial a_{n}}= & \frac{1}{\left(k_{j} \sum_{i} x_{i j}^{2}-\left(\sum_{i} x_{i j}\right)^{2}\right)^{2}} \\
& \cdot\left\{\left(k_{j} \sum_{i} x_{i j}^{2}-\left(\sum_{i} x_{i j}\right)^{2}\right)\right. \\
& \cdot\left[2 \sum_{i}\left(x_{i j} \frac{\partial x_{i j}}{\partial a_{n}}\right) \sum_{i} y_{i j}\right. \\
& +\sum_{i} x_{i j}^{2} \sum_{i} \frac{\partial y_{i j}}{\partial a_{n}} \\
& -\sum_{i} x_{i j} \sum_{i}\left(x_{i j} \frac{\partial y_{i j}}{\partial a_{n}}+y_{i j} \frac{\partial x_{i j}}{\partial a_{n}}\right) \\
& \left.-\sum_{i} \frac{\partial x_{i j}}{\partial a_{n}} \sum_{i} x_{i j} y_{i j}\right] \\
& -2\left(\sum_{i} x_{i j}^{2} \sum_{i} y_{i j}-\sum_{i} x_{i j} \sum_{i} x_{i j} y_{i j}\right) \\
& \left.\cdot\left[k_{j} \sum_{i}\left(x_{i j} \frac{\partial x_{i j}}{\partial a_{n}}\right)-\sum_{i} x_{i j} \sum_{i} \frac{\partial x_{i j}}{\partial a_{n}}\right]\right\}
\end{aligned}
$$

$$
\frac{\partial E}{\partial a_{n}}=\sum_{j=1}^{L}\left(\frac{1}{k_{j}} \sum_{i=1}^{k_{j}}\left|\frac{\left(1+b_{1 j}^{2}\right)\left(\frac{\partial b_{0 j}}{\partial a_{n}}+b_{1 j} \frac{\partial x_{i j}}{\partial a_{n}}+x_{i j} \frac{\partial b_{1 j}}{\partial a_{n}}\right)-\left(b_{0 j}+b_{1 j} x_{i j}-y_{i j}\right)\left(b_{1 j} \frac{\partial b_{1 j}}{\partial a_{n}}\right)}{\left(1+b_{1 j}^{2}\right)^{3 / 2}}\right|\right)
$$




$$
\begin{aligned}
\frac{\partial b_{1 j}}{\partial a_{n}}= & \frac{1}{\left(k_{j} \sum_{i} x_{i j}^{2}-\left(\sum_{i} x_{i j}\right)^{2}\right)^{2}} \\
& \cdot\left\{\left(k_{j} \sum_{i} x_{i j}^{2}-\left(\sum_{i} x_{i j}\right)^{2}\right)\right. \\
& \cdot\left[k_{j} \sum_{i}\left(y_{i j} \frac{\partial x_{i j}}{\partial a_{n}}\right)+x_{i j} \frac{\partial y_{i j}}{\partial a_{n}}\right) \\
& \left.-\sum_{i} x_{i j} \sum_{i} \frac{\partial y_{i j}}{\partial a_{n}}-\sum_{i} y_{i j} \sum_{i} \frac{\partial x_{i j}}{\partial a_{n}}\right] \\
& -2\left(k_{j} \sum_{i} x_{i j} y_{i j}-\sum_{i} x_{i j} \sum_{i} y_{i j}\right) \\
& \left.\cdot\left[k_{j} \sum_{i}\left(x_{i j} \frac{\partial x_{i j}}{\partial a_{n}}\right)-\sum_{i} x_{i j} \sum_{i} \frac{\partial x_{i j}}{\partial a_{n}}\right]\right\}
\end{aligned}
$$

In (15) and (16), $x_{i j}$ and $y_{i j}$ are calculated as

$$
\left[\begin{array}{l}
x_{i j} \\
y_{i j}
\end{array}\right]=\left[\begin{array}{l}
u_{c}^{\prime} \\
v_{c}^{\prime}
\end{array}\right]+\rho_{i j}(\Delta)\left[\begin{array}{c}
\cos \theta_{i j}^{\prime} \\
\sin \theta_{i j}^{\prime}
\end{array}\right]
$$

where $\rho_{i j}(\Delta)$ and $\theta_{i j}^{\prime}$ are the new magnitude and argument of the vector drawn from the distortion center to the new center of the test dot $P_{i j}$ after $\Delta$ th iteration. $\rho_{i j}(\Delta)$ can be computed as

$$
\rho_{i j}(\Delta)=\sum_{n=1}^{N} a_{n}(\Delta) \rho_{i j}^{\prime}
$$

$\partial x_{i j} / \partial a_{n}$ and $\partial y_{i j} / \partial a_{n}$ can be obtained from (17) and (18) as

$$
\frac{\partial x_{i j}}{\partial a_{n}}=\rho_{i j}^{\prime} \cos \theta_{i j}^{\prime}, \quad \frac{\partial y_{i j}}{\partial a_{n}}=\rho_{i j}^{\prime} \sin \theta_{i j}^{\prime} .
$$

The iterative procedure ends when the total error becomes smaller than a prespecified limit $\varepsilon$, i.e., $E(\Delta) \leq \varepsilon$. In the process of distortion correction $E$ decreases as the grid lines get straightened, and it reaches a minimum value $E_{\text {min }}$ when the most optimally straightened grid is obtained. If the image is expanded further, $E$ starts increasing. Thus, if $\varepsilon$ is chosen less than $E_{\text {min }}$, the diverging trend in total error is avoided by stopping the iterations further when $E(\Delta+1) \geq E(\Delta)$. The overall procedure for the estimation of the expansion coefficients is given in Fig. 2.

\section{B. Estimation of Back Mapping Polynomial Coefficients}

Once the expansion coefficients are computed, all the pixels contained in the distorted image space are mapped onto the corrected image space. It can be observed that a number of pixel locations are left vacant in the corrected image space, due to the inhomogeneous expansion of the distorted image. The expanded image in the corrected image space with vacant pixel positions is shown in Fig. 3. To obtain the correct intensity information of these vacant pixels, a back-mapping polynomial is derived which maps every pixel from the corrected image space onto the distorted image space. This polynomial is

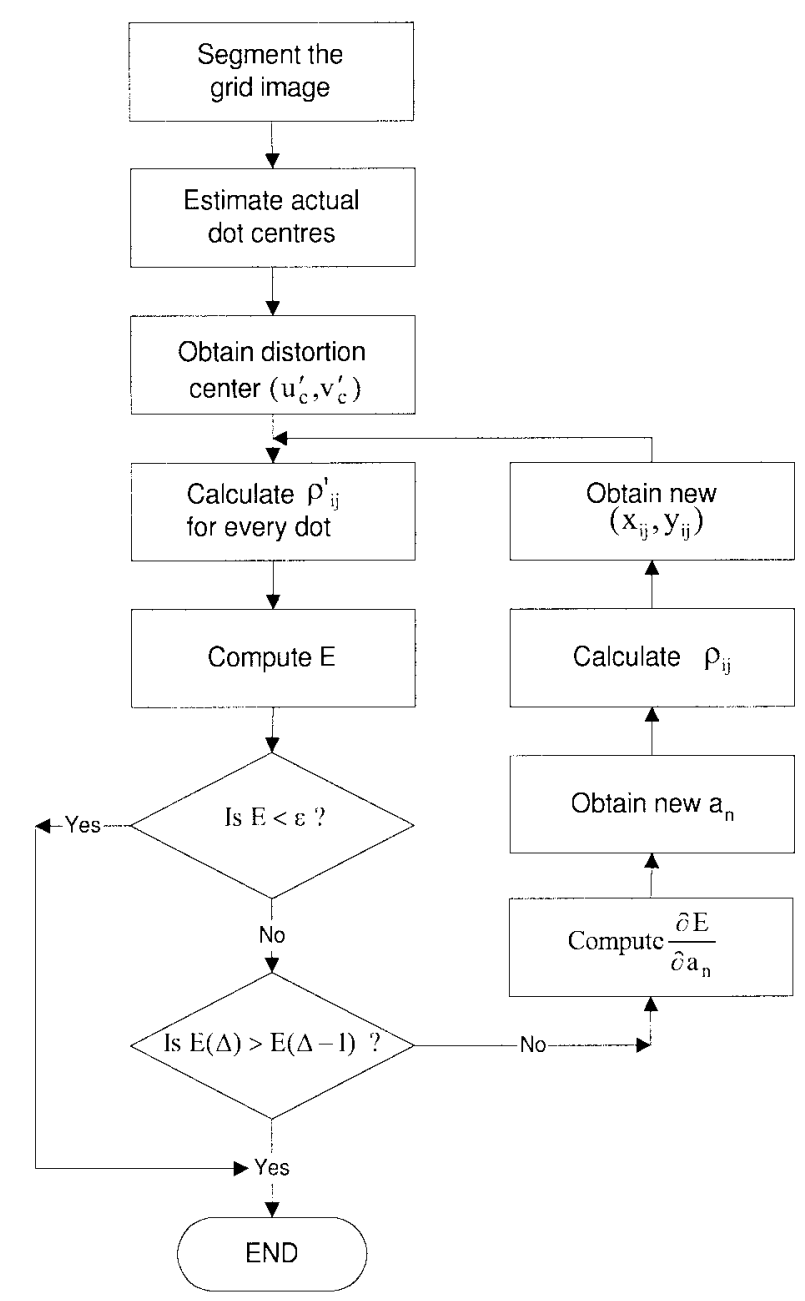

Fig. 2. Flow chart to estimate the expansion coefficients.

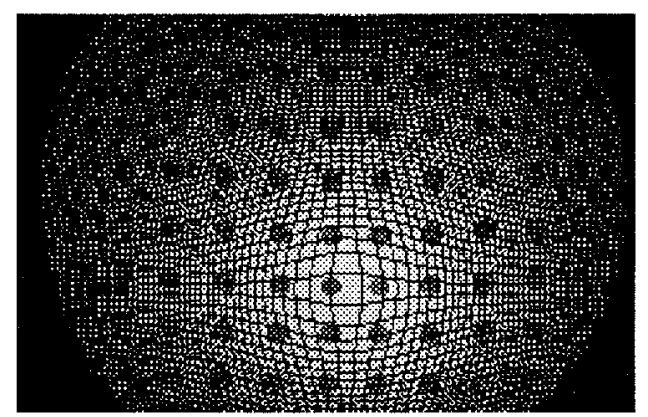

Fig. 3. Corrected image corresponding to Fig. 1 before back mapping.

defined in a way similar to the expansion polynomial of (3), and the coefficients are calculated by using nonlinear regression analysis employing least squares for a finite number of points in the distorted image [15]. For every pixel in the corrected image, the corresponding location in the distorted image is obtained and the information contained in that pixel location is assigned to the corrected image pixel. In case the pixel positions calculated using the back-mapping polynomial are nonintegers, a linear interpolation on the surrounding pixels is used to get the approximate pixel information. 


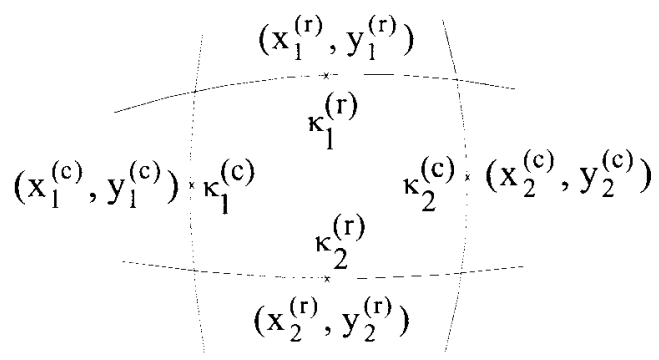

Fig. 4. Best fit polynomial curves of adjacent rows and columns of opposite curvatures.

\section{Estimation of Distortion Center and Corrected Center}

A reasonably correct estimation of the distortion center is essential for effective determination of the expansion coefficients. The distortion center is a fixed point for a particular camera and, once calculated, can be used for all the images obtained from that camera. From Fig. 1, it can be noted that the curvature of columns decreases from the first to the seventh column and then increases from the eighth to the thirteenth column, but the sign of the curvature changes. The same observation holds true for the rows also. Thus, the lines which remain straight after imaging must lie between the adjacent rows and columns of opposite curvatures. The rows and columns of opposite curvatures are given in Fig. 4. The intersection of such straight lines gives the distortion center of the image. In the proposed method of distortion center estimation, two polynomials, each of degree $\gamma$, passing through a set of grid dots of the adjacent rows of opposite curvatures are defined as

$$
q_{k}^{(r)}(x)=\sum_{l=0}^{\gamma} c_{k l}^{(r)} x^{l}, \quad \text { for } k=1,2
$$

The best fit polynomial coefficients $c_{k l}^{(r)}$ are obtained using an equation similar to (9). The curvature $\kappa_{k}^{(r)}$ of $k$ th row is computed at the stationary point on a curve, $\left(x_{k}^{(r)}, y_{k}^{(r)}\right)$ shown in Fig. 4 as

$$
\kappa_{k}^{(r)}=\frac{\left.\frac{d^{2} q_{k}^{(r)}(x)}{d x^{2}}\right|_{\left(x_{k}^{(r)}, y_{k}^{(r)}\right)}}{\left\{1+\left(\left.\frac{d q_{k}^{(r)}(x)}{d x}\right|_{\left(x_{k}^{(r)}, y_{k}^{(r)}\right)}\right)^{2}\right\}^{3 / 2}}, \quad \text { for } k=1,2 .
$$

Similar polynomials $q_{k}^{(c)}(x)$ for $k=1,2$ are also defined for the adjacent columns and the column curvatures $\kappa_{k}^{(c)}$ for the $k$ th column at the stationary point $\left(x_{k}^{(c)}, y_{k}^{(c)}\right)$ are obtained. The distortion center $\left(u_{c}^{\prime}, v_{c}^{\prime}\right)$ is estimated by interpolating the four curvatures $\kappa_{1}^{(r)}, \kappa_{2}^{(r)}, \kappa_{1}^{(c)}$, and $\kappa_{2}^{(c)}$ as

$$
\begin{aligned}
u_{c}^{\prime} & =\frac{\kappa_{1}^{(c)} x_{1}^{(c)}+\kappa_{2}^{(c)} x_{2}^{(c)}}{\kappa_{1}^{(c)}+\kappa_{2}^{(c)}} \\
v_{c}^{\prime} & =\frac{\kappa_{1}^{(r)} y_{1}^{(r)}+\kappa_{2}^{(r)} y_{2}^{(r)}}{\kappa_{1}^{(r)}+\kappa_{2}^{(r)}} .
\end{aligned}
$$

The corrected image center is needed for back mapping, as all the vectors $\boldsymbol{P}$ in the corrected image are obtained with respect to this center. To find this center a pixel location is computed in the distorted image which, after distortion correction, corresponds to the corrected image center. This pixel location is found based on the criterion that in the corrected image, pixel distances between the dot centers should be the same for all the grid lines in the horizontal and the vertical directions. The corrected image center is estimated by applying the expansion polynomial to this pixel location in the distorted image, which is obtained by iteratively minimizing the variation in distances between the test dot centers in the corrected image.

\section{EXTRACTION PROCEDURE TO OBTAIN DOT CENTERS OF THE EXPERIMENTAL GRID}

Qualitative results of the distortion-correction procedure depend upon the accurate estimation of centers of the grid dots in the distorted image, because the mathematical model uses these dot centers as its input. It has been observed that deviation in dot centers, even by a fraction of a pixel, introduces considerable errors during the computation of the expansion coefficients. To find the actual center for each dot lying in the $i$ th row and $j$ th column of the test grid, a new dualstep technique is proposed in which, at first, a pseudocenter is estimated by token matching, and then the actual center is calculated using integrated neighborhood search.

\section{A. Pseudocenter Estimation by Token Matching}

In this method, the darker regions representing the test dots in the grid image are segmented by identifying an appropriate threshold, using the dynamic histogram thresholding technique, and the image is binarized. The dynamic histogram thresholding is implemented by using the hillclustering approach to find a suitable valley as a threshold [16].

The pseudocenter is estimated as follows.

1) Define a token window $W$ of size $w \times w$ such that the intensity of the window pixel at location $(x, y), I^{\prime}(x, y)=$ $255 \forall(x, y) \in W$. An odd number is assigned to $w$ to ensure symmetry for token matching and is selected in accordance with the maximum dot size to allow full superposition of the dots by the window $W$.

2) For the captured image of size $L_{1} \times L_{2}$, for all the pixels belonging to the image region $((w-1 / 2),(w-1) / 2)) \times$ $\left.\left(L_{1}-(w-1 / 2), L_{2}-(w-1) / 2\right)\right)$, the window $W$ is superposed on each pixel $p_{x y}$ such that its center matches with the location $(x, y)$.

3) Compute choice value $C_{x y}$ for each pixel $p_{x y}$ as

$$
\begin{aligned}
C_{x y}= & \sum_{l=(y-(w-1 / 2))}^{(y+(w-1 / 2))} \sum_{k=(x-(w-1 / 2))}^{(x+(w-1 / 2))} \\
& \cdot\left(I^{\prime}\left(k+\frac{w-1}{2}-x, l+\frac{w-1}{2}-y\right)-I(k, l)\right)
\end{aligned}
$$

where $I(k, l)$ is the intensity of the pixel $p_{k l}$ of the grid image. 
TABLE I

Deviation of Actual Centers of the Test Dots Computed by an Integrated Neighborhood Search from the Pseudocenters Obtained by Token Matching

\begin{tabular}{|c|c|c|c|c|c|c|c|}
\hline Sl. No. & 1 & 2 & 3 & 4 & 5 & 6 & 7 \\
\hline $\begin{array}{l}\text { Pseudo } \\
\text { center }\end{array}$ & $\begin{array}{l}70.00 \\
45.00\end{array}$ & $\begin{array}{c}55.00 \\
5.00\end{array}$ & $\begin{array}{l}39.00 \\
32.00\end{array}$ & $\begin{array}{l}53.50 \\
45.50\end{array}$ & $\begin{array}{c}115.00 \\
46.00\end{array}$ & $\begin{array}{l}53.00 \\
30.00\end{array}$ & $\begin{array}{l}58.00 \\
97.50\end{array}$ \\
\hline $\begin{array}{l}\text { Actual } \\
\text { center }\end{array}$ & $\begin{array}{l}68.95 \\
46.15\end{array}$ & $\begin{array}{c}53.76 \\
6.07\end{array}$ & $\begin{array}{l}38.28 \\
32.53\end{array}$ & $\begin{array}{l}52.49 \\
46.51\end{array}$ & $\begin{array}{c}113.72 \\
46.45\end{array}$ & $\begin{array}{l}52.51 \\
31.49\end{array}$ & $\begin{array}{l}57.46, \\
98.51\end{array}$ \\
\hline $\begin{array}{l}\text { Difference } \\
\text { (Pixels) }\end{array}$ & $\begin{array}{l}1.05 \\
1.15\end{array}$ & $\begin{array}{l}1.24 \\
1.07\end{array}$ & $\begin{array}{l}0.72 \\
0.53\end{array}$ & $\begin{array}{l}1.01 \\
1.01\end{array}$ & $\begin{array}{l}1.28 \\
0.45\end{array}$ & $\begin{array}{l}0.49 \\
1.49\end{array}$ & $\begin{array}{c}0.64 \\
1.01\end{array}$ \\
\hline
\end{tabular}

4) Arrange $C_{x y}, \forall p_{x y}$ in descending order in a choice queue.

5) For the maximum $C_{x y}$ eliminate all the choice values $C_{m n}$ from the choice queue for which $|m-x| \leq \xi$ and $|n-y| \leq \xi$ where $\xi$ is a limiting number. $\xi$ is chosen in accordance with the size of dots and the distance between them to ensure that all the neighbor pixels $p_{m n}$ belong to the same test dot. In case one or more neighbor pixels contain the same choice value as that of pixel $p_{x y}$, a spatial average of all these pixels is used as the pixel location for $C_{x y}$ in the choice queue instead of $(x, y)$.

6) Take the next maximum value from the remaining choice queue and repeat step 5 .

7) Repeat step 6 until there is no neighbor pixel for all the pixels in the choice queue.

The locations of the pixels corresponding to the choice values in the final choice queue give the pseudocenters of the test dots.

\section{B. Region Identification by Integrated Neighborhood Search}

Once the pseudocenter for each of the test dots in the binarized image is obtained, the exact center is calculated by an integrated neighborhood search. In this procedure, the pseudocenter is used as a seed to identify all the pixels in the neighborhood region belonging to that particular test dot. Further, a search is performed on the eight pixels surrounding the pseudocenter. The black pixels are stored in an array called the neighborhood pixel array (NPA) while the white pixels are excluded from it. For each pixel contained in NPA, the surrounding pixels are checked and the pixels which are found black in the new search are again stored in NPA if they are not already present in it. This sequence is continued until all the pixels in the neighborhood region of the pseudocenter are exhausted. After identifying the full neighborhood region, the spatial center $\left(x_{i j}, y_{i j}\right)$, representing the actual dot center, is calculated by using all the pixels contained in NPA as

$$
x_{i j}=\frac{1}{K_{i j}} \sum_{l=1}^{K_{i j}} x_{i j}(l), \quad y_{i j}=\frac{1}{K_{i j}} \sum_{l=1}^{K_{i j}} y_{i j}(l)
$$

where $\left(x_{i j}(l), y_{i j}(l)\right)$ is the location of the $l$ th pixel in the NPA of the dot in the $i$ th row and $j$ th column of the test grid and $K_{i j}$ is the size of the NPA. Likewise, the actual centers of all the test dots in the grid image are calculated.

\section{EXPERIMENTAL RESULTS}

For the proposed distortion-correction procedure, an experimental grid containing a rectangular array of dots of 1-mm diameter was used. The distance between the dot centers was chosen as $2.5 \mathrm{~mm}$ in horizontal as well as vertical directions. The small distance between the dots was used in order to facilitate imaging of a large number of dots from the endoscope. This is preferred to enhance the accuracy of the model parameter estimation. An electronic videoendoscopy system (Fujinon), which utilizes a CCD camera with 200000 -pixel resolution and three light sources, red, green, and blue, was used for capturing the images. The captured images were digitized by a frame grabber and stored in an image buffer. The grid was attached to a copy-stand platform and the camera of the endoscope was oriented perpendicular to the grid surface at a distance of $10 \mathrm{~mm}$. After imaging, as shown in Fig. 1, it was observed that the distance between the test dot centers decreases as they move away from the distortion center. This implies that the distortion-correction procedure must produce an image in which distances between grid dot centers away from the distortion center are approximately equal to those which are close to the center.

The digitized image of size $144 \times 110$ pixels was binarized using the histogram thresholding approach by considering the second valley of the histogram as the threshold point. A total of 58 dots were extracted for the distortion-correction model formulation. To estimate the pseudocenters of the test dots by token matching, a window $W$ of size $5 \times 5$ pixels was used. The limiting number $\xi$ was chosen as six, as it conforms with the dot size and the distance between the dot centers. The actual centers were calculated by using an integrated neighborhood search. The deviation of the actual center from the corresponding pseudocenter for seven test dots is shown in Table I. It can be observed that the deviation is less than two pixels. It was found that this error was enough to introduce significant degradations in the distortion-correction results.

Before computing the expansion polynomial it is necessary to compute the distortion center of the endoscopic image. The 


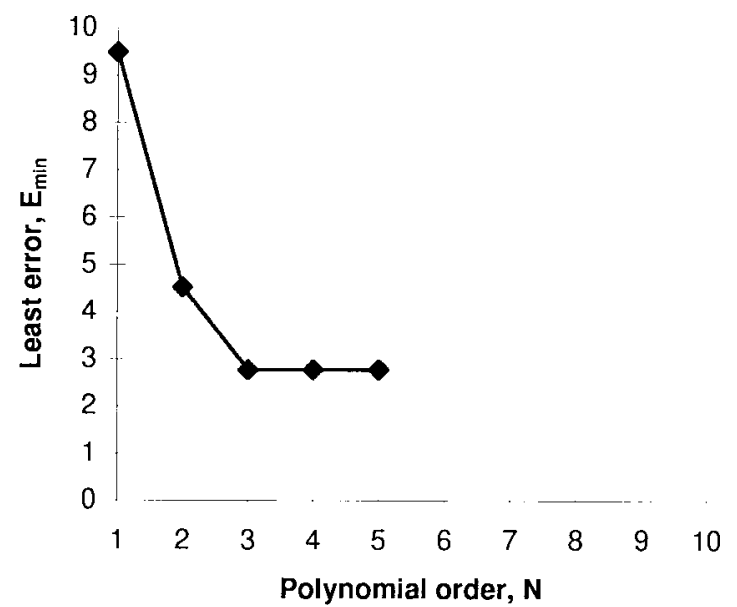

Fig. 5. Variation in least total error $\left(E_{\min }\right)$ with respect to the order of expansion polynomial.

distortion center was estimated by using curvature criterion and was found to be $(69,62)$ while the coordinate system was fixed at the bottom left corner of the image. To compensate for unavoidable errors, a small region (a window of size $5 \times 5$ pixels) around the estimated distortion center was selected, and a pixel lying within that window was chosen as the distortion center for which the total error $E$ is minimum. To obtain the expansion polynomial, first of all the degree of polynomial was ascertained. For this purpose, the relationship between the degree of polynomial and the least total error Emin was examined experimentally, and it was found that the variation in error was negligible for the polynomial of order more than three, as shown in Fig. 5. We have chosen the order of expansion polynomial as four. The four expansion coefficients were obtained by using (9)-(19). The expansion coefficients were computed from the iterative relation given in (12) for different values of the convergence rate parameter $\alpha$. The value of the expansion index $\beta$ was found to be optimum at 2.3 , which decides the weight of the $n$th coefficient. The rate of convergence of total error for two different values of $\alpha$ is shown in Fig. 6. It can be observed that as $\alpha$ increases, convergence becomes faster. The number of iterations required for convergence and the corresponding $E_{\min }$ for different values of $\alpha$ are given in Table II. As $\alpha$ decreases, $E_{\min }$ reduces while the number of iterations required for convergence increases. But $E_{\min }$ does not decrease significantly after a particular value of $\alpha, \alpha_{\mathrm{opt}}$, though the number of iterations required for convergence increases exponentially. Hence, to restrict the computation time to make it suitable for online camera calibration, the value of $\alpha$ was chosen as a tradeoff between the accuracy and the computation time. In the present experimental setup, $\alpha_{\text {opt }}$ was chosen as 0.005 . For the set of expansion coefficients obtained, the relationship between the distorted radius and the corresponding corrected radius is shown in Fig. 7. It can be observed that the outer areas of the distorted image having large radii expand more after distortion correction. The mean error, representing deviation of the dot centers from the best fit straight line before and after distortion correction for typical dot columns in the grid image shown in Fig. 1, is given in Table III.
The back-mapping coefficients are found by using a finite number of image dots. For back mapping, the pixel location in the distorted image corresponding to the corrected center was found as $(74,45)$. The corrected image is shown in Fig. 8. It can be seen that the image gets magnified after correction and the grid is straightened within practical limits. The magnification preserves the information around the distortion center. In the corrected image the dot centers deviate slightly from the theoretical straight line because of the initial assumption of purely radial distortion. Minor errors in the estimation of the dot centers and the interpolation of neighborhood pixels to find the information contained in the noninteger pixel locations also affect the quality of the corrected image. According to the implementation of the proposed method on a PC (Pentium $166 \mathrm{MHz}$ ), it is found that with $\alpha=0.005$ and $\beta=2.3$ the algorithm converged in 22 iterations (5.95 s). At the same time, the implementation of the Hideaki's method [12] on the same platform with the most appropriate selected set of coefficients $\varepsilon_{k}:\{0.0001,0.0000001,0.0000000001\}$ for a polynomial of order four $(k=3)$ needed 116 iterations $(28.17 \mathrm{~s})$ for convergence. Hence, it can be seen that there is a considerable improvement in the computational speed for the distortioncorrection procedure by using the proposed technique.

In the present model, calculation of the model parameters is independent of the orientation of the test grid. To verify the proposed model, a grid image captured by tilting the experimental grid both in the horizontal as well as vertical planes, as shown in Fig. 9, is corrected using the same expansion polynomial. The corrected image is shown in Fig. 10. It can be seen from the corrected image that the computed polynomial is capable of correcting the distortion in the image captured in any orientation. The same expansion polynomial can be used for distortion correction of the images taken by the videoendoscope until the time the camera lens is not changed or relocated from the CCD array. According to the physics of endoscopic imaging, as the distance between the grid and the camera increases, the image distortion decreases. Typical endoscopic images are taken within a range of less than 20 $\mathrm{mm}$ during clinical procedures. The same distortion-correction polynomial was applied to several test images captured within a viewing range of $20 \mathrm{~mm}$, and it was observed that the corrected images were acceptable for further analysis.

\section{DISCUSSION}

While performing endoscopy it is important for the endoscopist to know the approximate size of the ulcers and abnormal growths. It is necessary not only for identification of the status of the gastrointestinal tract but also to know the severity of the probable disease. Due to nonlinear distortion, the outer areas of captured endoscopic images are compressed more than the inner areas, thus seriously undermining the inferential capability of an endoscopist to determine the probable size of the region of interest. While analyzing the endoscopic images automatically, by employing software algorithms based on artificial intelligence, distortion becomes a bottleneck for the spatial quantitative parameter 


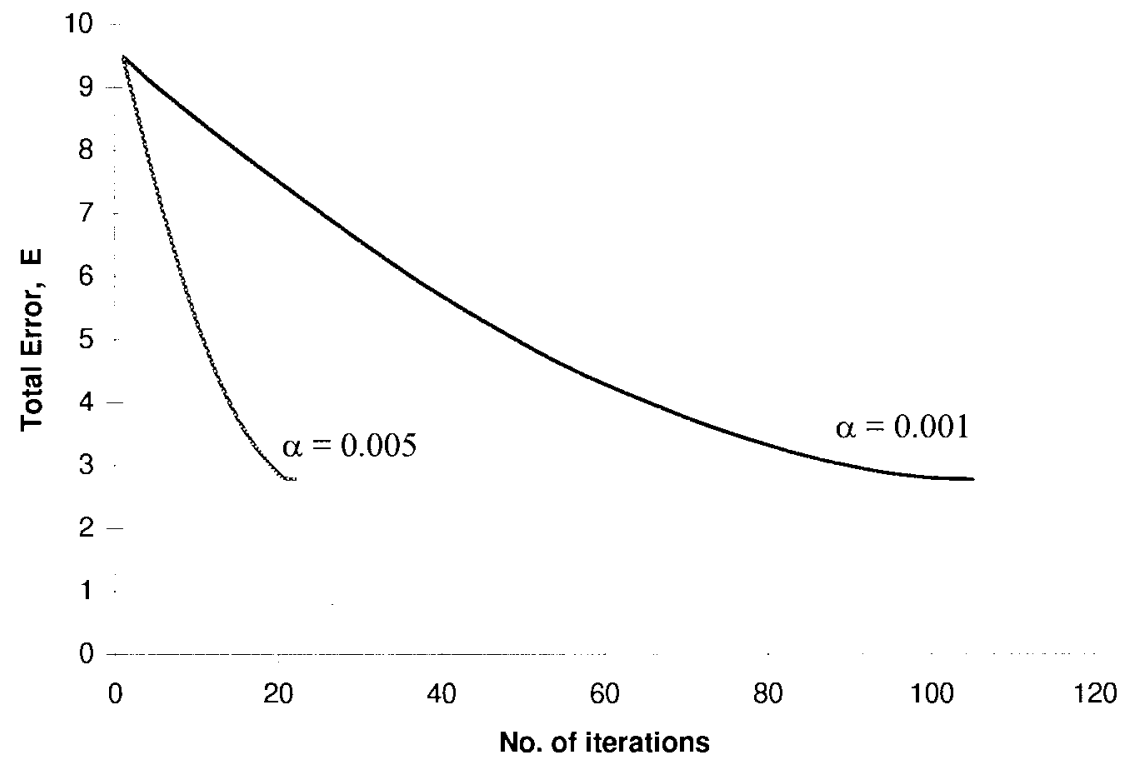

Fig. 6. Rate of convergence of the total error for different values of $\alpha$.

TABLE II

Number of Iterations ReQuired for Convergence and the Respective Least Total ERrors for Different Values of $\alpha$

\begin{tabular}{c|c|c|c|c|c|c|c}
\hline$\alpha$ & 0.1000 & 0.0500 & 0.0100 & 0.0050 & 0.0010 & 0.0005 & 0.0001 \\
\hline $\begin{array}{c}\text { Least total error } \\
\left(E_{\min }\right)\end{array}$ & 3.6015 & 2.9573 & 2.8057 & 2.7843 & 2.7793 & 2.7788 & 2.7784 \\
\hline No. of iterations & 2 & 3 & 12 & 22 & 104 & 209 & 1036 \\
\hline
\end{tabular}

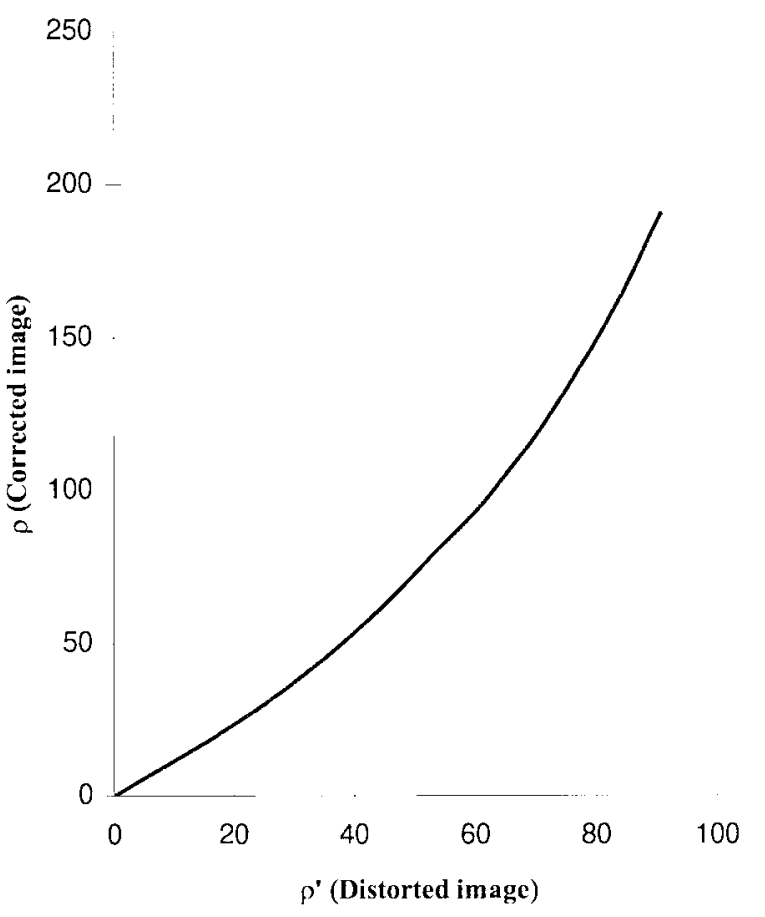

Fig. 7. Relationship between the vector magnitudes before and after distortion correction. extraction. That is why a corrective procedure to eliminate the distortion of the endoscopic image is absolutely essential.

To demonstrate the effects of the proposed distortioncorrection technique, several gastrointestinal images were captured and corrected by using the same expansion polynomial. A typical gastrointestinal image is shown in Fig. 11 and the corresponding corrected image is shown in Fig. 12. Similarly, a second distorted image and its corrected version are given in Figs. 13 and 14, respectively. It can be noted that the original images were magnified and outer areas expanded considerably due to the distortion correction. The corrected images were seen by the expert endoscopists and the opinions expressed were quite favorable for the visual observation of the internal structure of the tract. In addition, the quantitative parameters extracted from the corrected images were suitable for predicting the status of the gastrointestinal region, by using artificial intelligence.

\section{CONCLUSION}

A novel method for correcting the nonlinear distortion in endoscopic images has been proposed. The new method requires a simple procedure, as it is independent of the orientation of the test grid. The expansion coefficients were obtained by using least squares estimation and were applied 
TABLE III

Mean Error (Deviation of the Dot Centers from the Best Fit Straight Line in PiXels) for Typical Dot Columns in the Grid Image Shown in Fig. 1

\begin{tabular}{c|c|c|c|c|c|c|c}
\hline & Col. 1 & Col. 2 & Col. 3 & Col. 4 & Col. 5 & Col. 6 & Col. 7 \\
\hline $\begin{array}{c}\text { Mean error } \\
\text { (before correction) }\end{array}$ & 2.72 & 1.57 & 0.89 & 0.19 & 0.72 & 1.13 & 2.26 \\
\hline $\begin{array}{c}\text { Mean error } \\
\text { (after correction) }\end{array}$ & 0.34 & 0.32 & 0.27 & 0.18 & 0.25 & 0.30 & 0.33 \\
\hline
\end{tabular}

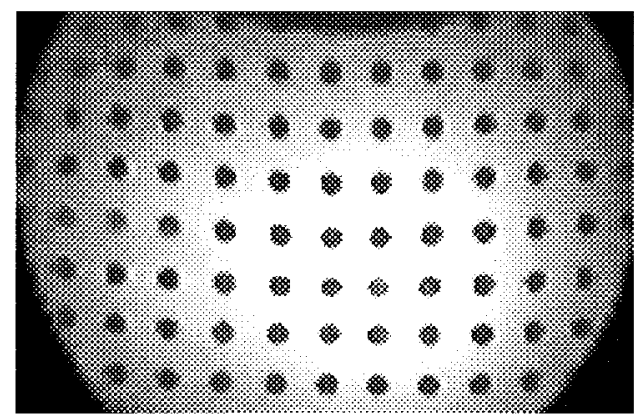

Fig. 8. Corrected image corresponding to Fig. 1 after back mapping.

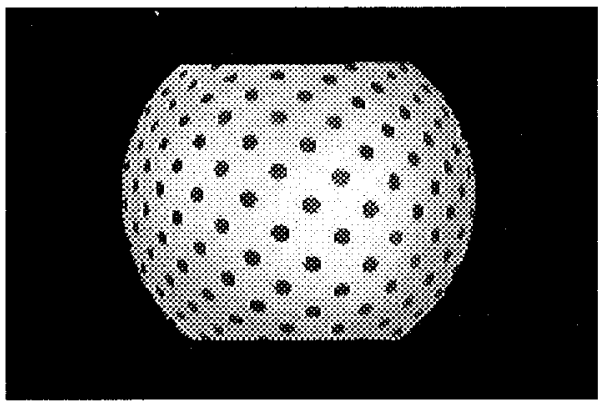

Fig. 9. Distorted image of a grid tilted in horizontal as well as vertical directions.

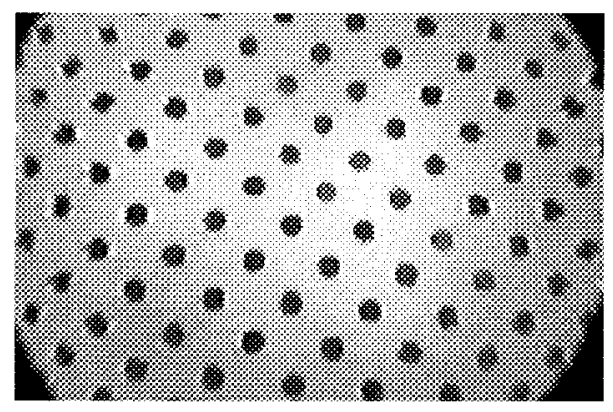

Fig. 10. Corrected image corresponding to Fig. 9.

to different grid patterns. It was observed that the expansion polynomial obtained for a particular endoscope camera lens was capable of correcting the distortion satisfactorily. This procedure is an essential step for the accurate measurement of

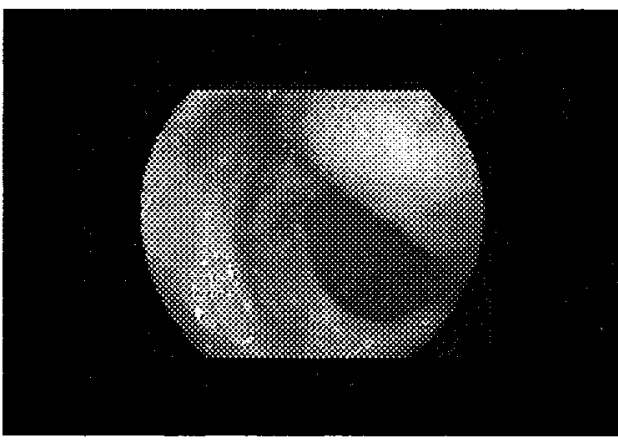

Fig. 11. A typical gastrointestinal image captured by an endoscope.

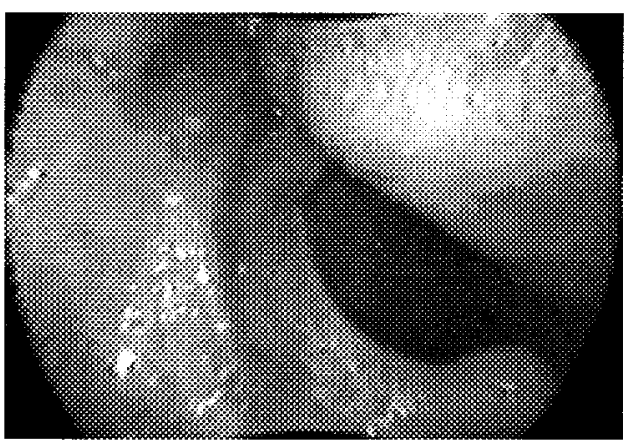

Fig. 12. Corrected image corresponding to Fig. 11.

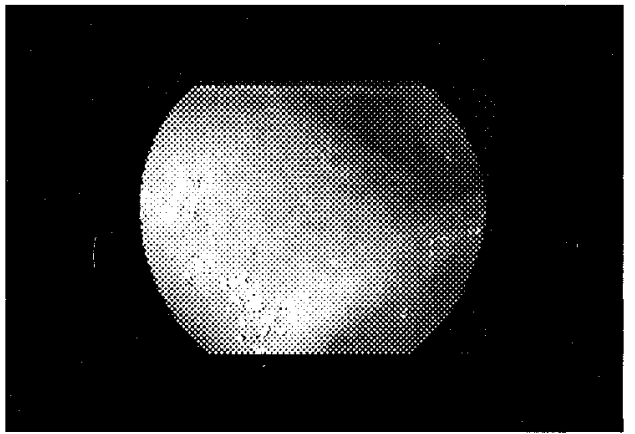

Fig. 13. Gastrointestinal image before distortion correction.

the regions of interest in the endoscopic images to facilitate quantitative parameter extraction for decision making. Further 


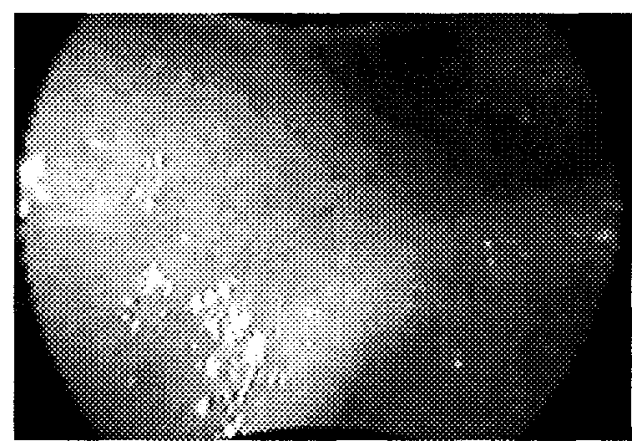

Fig. 14. Corrected image corresponding to Fig. 13.

research is in progress to find a relationship between the expansion coefficients and the distance of the camera lens from the object. The high-speed response of the proposed algorithm makes the concept of online camera calibration feasible.

\section{REFERENCES}

[1] H. Kato and J. P. Barron, Electronic Videoendoscopy. Switzerland: Harwood, 1993.

[2] A. Sonnenberg, M. Giger, L. Kern, C. Noll, K. Stuby, K. B. Weber, and A. L. Blum, "How reliable is determination of ulcer size by endoscopy," Brit. Med. J., vol. 24, pp. 1322-1324, 1979.

[3] Y. Hatada, S. Iwane, H. Tohno, T. Baba, A. Munakata, and Y. Yoshida, "A new method for the measurement of the gastric and colonic lesions with an electronic endoscope and image processor," Gastrointestinal Endoscopy, vol. 37, p. 275, 1991.
[4] C. Margulies, B. Krevsky, and M. F. Catalano, "How accurate are endoscopic estimates of size," Gastrointestinal Endoscopy, vol. 40, pp. 174-177, 1994

[5] Q. MaoLin and S. De Ma, "Parametric and nonparametric approaches for camera calibration: Analysis of imaging errors and their compensation," in Proc. Sec. Asian Conf. Computer Vision, Singapore, 1995, pp. II 205-II 209.

[6] M. Li and L. Jean-Marc, "Some aspects of zoom lens camera calibration," IEEE Trans. Pattern Anal. Machine Intell., vol. 18, pp. 1105-1110, Nov. 1996

[7] R. Y. Tsai, "An efficient and accurate camera calibration technique for 3D machine vision," in Proc. IEEE Computer Vision Pattern Recognition, Miami, FL, June 1986, pp. 364-374.

[8] D. C. Brown, "Decentering distortion of lenses," Photogramm. Eng. Remote Sensing, May 1966, pp. 444-462.

[9] Y. Nomura, M. Sagara, H. Naruse, and A. Ide, "Simple calibration algorithm for high-distortion-lens camera," IEEE Trans. Pattern Anal. Machine Intell, vol. 14, pp. 1095-1099, Nov. 1992.

[10] J. Weng, P. Cohen, and M. Herniou, "Camera calibration with distortion models and accuracy evaluation," IEEE Trans. Pattern Anal. Machine Intell., vol. 14, pp. 965-980, Oct. 1992.

[11] W. E. Smith, N. Vakil, and S. A. Maislin, "Correction of distortion in endoscopic images," IEEE Trans. Med. Imag., vol. 11, pp. 117-122, Mar. 1992.

[12] H. Hideaki, Y. Yagihashi, and Y. Miyake, "A new method for distortion correction of electronic endoscope images," IEEE Trans. Med. Imaging, vol. 14, pp. 548-555, Sept. 1995.

[13] L. V. Atkinson and P. J. Harley, An Introduction to Numerical Methods with Pascal. London, U.K.: Addison-Wesley, 1983.

[14] S. G. Nash and A. Sofer, Linear and Nonlinear Programming. Singapore: McGraw-Hill, 1996, p. 312.

[15] D. A. Berry and B. W. Lindgren, Statistics: Theory and Methods. CA: Brooks/Cole, 1990.

[16] S. M. Krishnan, K. V. Asari, C. J. Yap, and P. M. Y. Goh, "Quantitative characterization of colon by fuzzy based image analysis," in Proc. ISIRS 98, Jan. 1998, pp. 53-55. 